\title{
Praxisnahe Qualitätskontrolle
}

\author{
Luise Menzi \\ Dr. phil., Leitung Rehabilitation, ANQ, Nationaler Verein für Qualitätsentwicklung in Spitälern und Kliniken
}

Qualitätsmessungen in Spitälern und Kliniken sind zwar gesetzlich vorgeschrieben, stossen aber nicht nur auf Zustimmung. Auf den ersten Blick binden sie Ressourcen. Auf längere Sicht zahlen sie sich jedoch aus. Sinnvolle Datenerhebungen und faire Betriebsvergleiche erfordern Zeit und Expertenwissen. Seit sechs Jahren setzt sich der ANQ dafür ein. Erste Ergebnisse sind sichtbar. Der Weg dahin am Beispiel der Rehabilitation.

Der ANQ führt im Auftrag der Tarifpartner landesweit einheitliche Qualitätsmessungen im stationären Bereich der Akutsomatik, der Rehabilitation und der Psychiatrie durch. Er erstellt national vergleichende Auswertungen und publiziert die Messresultate transparent, sobald die Datenqualität für faire Betriebsvergleiche ausreicht. Mit seiner Arbeit fördert der ANQ laufende Verbesserungsprozesse und stösst, wo notwendig, neue an.

\section{Initiative des Gesundheitswesens}

Das Krankenversicherungsgesetz (KVG) von 1994 bildet die Grundlage der ANQ-Tätigkeit. Es schreibt den Spitälern, Kliniken, Versicherern und Kantonen eine vertraglich vereinbarte Qualitätssicherung vor: Wirksamkeit, Zweckmässigkeit und Wirtschaftlichkeit der erbrachten Leistungen müssen periodisch überprüft werden. Dabei sind auch Spital- und Klinikvergleiche, insbesondere zu Kosten und medizinischer Ergebnisqualität, vorzunehmen. Wie sie den Qualitätsnachweis in der Praxis erbringen, wurde den Leistungserbringern und Kostenträgern überlassen. Zu diesem Zweck gründeten der Spitalverband $\mathrm{H}+$, der Branchenverband der Krankenversicherer santésuisse, alle Kantone und das Fürstentum Liechtenstein, die Schweizerische Gesundheitsdirektorenkonferenz und die Eidgenössischen Sozialversicherer (Unfall-, Militär- und Invalidenversicherung) 2009 den Verein ANQ. Das Bundesamt für Gesundheit (BAG) nahm als Beobachter Einsitz. Die aktuellen ANQ-Qualitätsmessungen sind den Spitälern und Kliniken also nicht auferlegt, sondern wurden von ihnen selbst initiiert. ANQ-Mitglieder respektive deren ausgewiesene Fachexpertinnen und -experten entwickeln und gestalten sämtliche Messungen und Prozesse. Dabei verfolgen sie einen praktischen An- satz: In der Rehabilitation beispielsweise werden die ANQ-Messungen direkt in den Behandlungsverlauf integriert. Es gilt, Daten und Zielsetzungen zu generieren, die unmittelbar angewendet werden können. Die Erhebungen laufen Hand in Hand mit dem Behandlungsprozess. Damit können die Messdaten aktiv genutzt, nicht nur theoretisch verglichen und archiviert werden.

\section{Landesweit anerkannte Messthemen}

Im ANQ-Messplan sind die Messthemen der einzelnen Fachbereiche festgelegt. Er ist in der Branche breit abgestützt und national anerkannt. Der Messplan für die Rehabilitation beruht auf den Erfahrungen und Ergebnissen der Pilotprojekte in der muskuloskelettalen und der neurologischen Rehabilitation (2006 bis 2011). Im Jahre 2013 wurde er national eingeführt, nahezu alle Schweizer Rehabilitationskliniken beteiligen sich inzwischen an den ANQ-Messungen. Ein ständiges Expertengremium, der Qualitätsausschuss (QA) Rehabilitation, begleitet und entwickelt den Messplan laufend weiter. Dabei bezieht der QA auch Rückmeldungen aus den Kliniken ein.

\section{Patientenorientiertes Vorgehen}

Bei den Qualitätserhebungen in allen Bereichen der Rehabilitation steht die Individualität der Patientin und des Patienten im Vordergrund. Zum Einsatz kommen, je nach Bereich, unterschiedliche Messinstrumente. In der muskuloskelettalen und in der neurologischen Rehabilitation werden bei Ein- und Austritt Assessments durchgeführt, um die Verbesserung der Funktionsfähigkeit der Patientin, des Patienten abzubilden. Dabei ist die Selbsteinschätzung der zu Behandelnden 
wie auch die Fremdbeurteilung durch das Rehabilitationsteam gefragt. Rehabilitationsziele werden gemeinsam mit den Patientinnen und Patienten festgelegt, deren Erreichung mit ihnen überprüft. Dies in enger Anlehnung an die WHO-Klassifikation ICF (International Classification of Functionality).

Dieses Vorgehen entspricht der heute gängigen Praxis in der Rehabilitation, bei der Patientinnen und Patienten bestmöglich in die Behandlungsplanung integriert werden und damit ihren Teil der Verantwortung für den Heilungsprozess übernehmen. Praktisch bedeutet das: Behandelnde und Patientin oder Patient erarbeiten zu Beginn, welches (Haupt-)Ziel während des Auf-

\section{Läuft alles nach Plan, wird im Sommer 2016 der erste nationale Vergleichsbericht für den Bereich Rehabilitation vorliegen.}

enthalts erreicht werden kann. Beispielsweise, ob sie wieder selbstständig wohnen, die Erwerbsarbeit wieder in Teilzeit oder Vollzeit aufnehmen werden usw. Dieses (Haupt-)Partizipationsziel und dessen Erreichen wird in den ANQ-Messungen abgefragt, weil es den roten Faden der Behandlung bildet. Dementsprechend können bei einer weiteren Messung auch Fragen wie «Können Sie einen Milchkarton (Tetrapack) öffnen?» gestellt werden, die auf den ersten Blick unangebracht erscheinen mögen. Für die Bewältigung des Alltags aber sind sie zentral.

In der kardiologischen und in der pneumologischen Rehabilitation werden die funktionale Einschränkung und deren Entwicklung über den Behandlungsprozess ebenfalls mit dafür geeigneten Messinstrumenten beurteilt. Gleichzeitig prüft man die Wahrnehmung der Patientin, des Patienten.

\section{Datenerhebung zugunsten fairer Spital- und Klinikvergleiche}

Mit dem Instrument CIRS (Cumulative Illness Rating Scale), einer Skala zur kumulierten Bewertung von Erkrankungen, werden in allen Bereichen der Rehabilitation weitere Patientendaten abgefragt. Die Klinik übermittelt dem ANQ zudem BFS-Daten der medizinischen Statistik. Der Grund: Um faire Spital- und Klinikvergleiche zu ermöglichen, müssen die Messresultate zuvor risikobereinigt werden. Dabei sind Faktoren herauszufiltern, die das Messergebnis negativ oder positiv beeinträchtigen, von den Institutionen jedoch nicht beeinflusst werden können. Ein Beispiel: Die Klientel einer Klinik ist bei Eintritt schwerer beeinträchtigt oder weist mehr Multimorbidität auf als die Klientel einer anderen Klinik. Ohne Risikobereinigung würde die erste Klinik im Qualitätsvergleich mit der zweiten schlechter abschneiden, auch wenn sie ein besseres Behandlungsergebnis erzielt.

\section{Erste greifbare Resultate}

Im Bereich Akutsomatik veröffentlicht der ANQ seit 2012 pseudonymisierte und seit 2014 spitalindividuelle Messresultate, beispielsweise die Wundinfektionsraten nach bestimmten chirurgischen Eingriffen. In der Psychiatrie werden die Ergebnisse seit 2013 zusammenfassend und die Symptombelastung in der Erwachsenenpsychiatrie voraussichtlich ab Herbst 2015 pro Klinik ausgewiesen. Die Publikation der Ergebnisqualität ermöglicht eine transparente und nationale Vergleichbarkeit. Spitäler und Kliniken können aufgrund dieser Erkenntnisse gezielt Massnahmen zur Verbesserung ihrer Qualität ergreifen.

\section{Aufbauphase abgeschlossen}

Im jüngsten ANQ-Messbereich, der Rehabilitation, wurde in den letzten beiden Jahren viel Aufbauarbeit geleistet: Kliniken implementierten die ANQ-Messungen, die gelieferte Datenqualität wurde laufend geprüft und Schritt für Schritt gesteigert. Denn erst eine ausreichende Datenqualität macht eine seriöse Auswertung möglich. Die Einführungsphase hatte noch keine sichtbaren Ergebnisse gezeigt und bot deshalb auch Anlass zu Kritik. Es liegt jedoch in der Natur der Aufgabe, dass die Einführung einheitlicher Messsysteme eine gewisse Vorlaufzeit benötigt. Diese Zeit war notwendig und wertvoll, um gemeinsam mit den Kliniken eine sorgfältige und praxisnahe Erfassung und Dokumentation zu erarbeiten.

Der Aufwand hat sich gelohnt: Im Sommer 2015 stellte sich heraus, dass die Datenqualität der Rehabilitationskliniken ein Niveau erreicht hat, das eine erste national vergleichende Auswertung des Datenjahrs 2014 zulässt. Aktuell definiert der QA die Analysekategorien für diese Auswertung. Läuft alles nach Plan, wird im Sommer 2016 der erste nationale Vergleichsbericht für den Bereich Rehabilitation vorliegen. Spätestens danach werden sich die ANQ-Messungen in allen Kliniken als integraler Teil des Therapieprozesses etabliert haben.

Disclosure statement

ANQ-Mitglieder: Spitalverband H+, santésuisse, Eidgenössische Sozialversicherer, alle Kantone und das Fürstentum Liechtenstein, Schweizerische Gesundheitsdirektorenkonferenz 